\title{
The helically-reduced wave equation as a symmetric-positive system
}

\author{
C. G. Torre \\ Department of Physics, Utah State University, Logan, Utah 84322-4415
}

(Received 9 June 2003; accepted 28 August 2003)

Motivated by the partial differential equations of mixed type that arise in the reduction of the Einstein equations by a helical Killing vector field, we consider a boundary value problem for the helically-reduced wave equation with an arbitrary source in 2+1 dimensional Minkowski space-time. The reduced equation is a second-order partial differential equation which is elliptic in a disk and hyperbolic outside the disk. We show that the reduced equation can be cast into symmetricpositive form. Using results from the theory of symmetric-positive differential equations, we show that this form of the helically-reduced wave equation admits unique, strong solutions for a class of boundary conditions which include Sommerfeld conditions at the outer boundary. (C) 2003 American Institute of Physics.

[DOI: 10.1063/1.1623930]

\section{INTRODUCTION}

Physical systems are typically governed by partial differential equations (PDEs) of a fixed type: elliptic, hyperbolic, or parabolic. The mathematical properties of such equations have been extensively investigated (see, e.g., Refs. 1 and 2). Considerably less is known about PDEs of mixed type, by which we mean equations whose type is different in different subdomains of the domain of interest, e.g., elliptic in one region and hyperbolic in another. ${ }^{3}$ Compared to elliptic, hyperbolic, or parabolic equations, mixed type equations are rather unusual, both in the boundary conditions that can be imposed to get existence and uniqueness of solutions as well as in the regularity of solutions that are obtained. Moreover, the lower-order terms in equations of mixed type take on a more significant role than in equations of fixed type. This latter feature means that it is difficult to obtain general results about PDEs of mixed type; to a large extent, one must investigate each set of equations, each set of boundary conditions, etc., separately.

In relativistic field theory on a fixed space-time, mixed type equations occur after performing a symmetry reduction of hyperbolic PDEs with respect to an isometry group which has an infinitesimal generator that changes type from timelike to spacelike. In generally covariant theories, such symmetry reductions may yield PDEs of mixed type in appropriate gauges. An important example of the latter type, currently of considerable interest in gravitational physics, arises in the quasi-stationary approximation to the two-body problem in general relativity. ${ }^{4-6}$ Here one is interested in solving the Einstein equations for space-times admitting a helical Killing vector field. The helical Killing vector field, which represents a rotating reference frame, will be timelike near the bodies and spacelike far from the bodies. The reduced Einstein equations (modulo gauge) can be expected to include nonlinear PDEs of mixed type on the $2+1$ dimensional manifold of orbits of the Killing vector field. The reduced equations can only be solved numerically, but one naturally desires as much a priori information about existence and uniqueness of solutions, regularity of solutions, admissible boundary conditions, etc. as one can get. The quasi-stationary approximation to the relativistic two-body problem is the principal motivation behind the work presented here.

As a warm-up for numerically solving the Einstein equations in the quasi-stationary approximation, the wave equation and some of its nonlinear extensions, reduced by the assumption of helical symmetry, have been examined, both analytically and numerically in Ref. 7. The helically- 
reduced wave operator is elliptic inside a "light cylinder" and hyperbolic outside the cylinder. It was shown in Ref. 7 that one can give a formal series solution of the helically-reduced wave equation for a source consisting of a pair of equal and opposite point charges, placed symmetrically with respect to the axis of helical symmetry. For these analytical solutions, Sommerfeld conditions were prescribed at an outer boundary (which may be at infinity). ${ }^{8}$ The choice of such boundary conditions can be motivated on physical grounds, and the apparent analytic existence of unique solutions in the linear case and numerical solutions in the nonlinear case gives confidence that the helically-reduced equation can be treated as a boundary value problem. However, from a mathematical point of view it is not immediately clear a priori why such boundary conditions are admissible, that is, why one should expect unique solutions to exist. Our goal here is to understand existence, uniqueness, regularity, etc.-in short, the well-posed nature of this problem-from a general point of view that does not rely upon explicitly constructing a solution to the PDE. The idea is that such a point of view can be used to better understand the helically-reduced Einstein equations, which will not yield so easily to a direct assault.

In the past, certain boundary value problems of mixed type have been addressed using the theory of "symmetric-positive" differential equations," which can be viewed as a generalization of

elliptic and hyperbolic equations. Friedrichs ${ }^{9}$ and Lax and Phillips ${ }^{10}$ have given the basic existence and uniqueness results for linear symmetric-positive equations. In this article we shall show that the helically-reduced wave equation with arbitrary sources in $2+1$ dimensions can be cast into symmetric-positive form. We can then deduce existence and uniqueness results for a class of boundary conditions that include the Sommerfeld conditions used in Ref. 7. These results provide support for the proposition that boundary value problems of mixed type arising from helical symmetry reductions - such as arise in the relativistic two-body problem-are well-posed.

\section{THE HELICALLY-REDUCED WAVE EQUATION}

We consider $2+1$ dimensional Minkowski space-time $\left(\mathbf{R}^{3}, \eta\right)$ and a helical Killing vector field $\xi$. There will exist an inertial-Cartesian coordinate chart $(t, x, y)$, such that

$$
\eta=-d t \otimes d t+d x \otimes d x+d y \otimes d y,
$$

and

$$
\xi=\frac{\partial}{\partial t}+\Omega\left(x \frac{\partial}{\partial y}-y \frac{\partial}{\partial x}\right)
$$

for some constant $\Omega$. In the corresponding inertial-polar coordinates $(t, r, \phi)$ we have

$$
\eta=-d t \otimes d t+d r \otimes d r+r^{2} d \phi \otimes d \phi,
$$

and

$$
\xi=\frac{\partial}{\partial t}+\Omega \frac{\partial}{\partial \phi} .
$$

Note that $\xi$ is not of a fixed type:

$$
\eta(\xi, \xi)=r^{2} \Omega^{2}-1 \begin{cases}>0 & \text { for } r>\frac{1}{\Omega}, \\ =0 & \text { for } r=\frac{1}{\Omega}, \\ <0 & \text { for } r<\frac{1}{\Omega} .\end{cases}
$$


We will call the set of space-time events with $r=1 / \Omega$ the light cylinder.

The wave equation with source $j: \mathbf{R}^{3} \rightarrow \mathbf{R}$ is given by

$$
\square \Phi=j
$$

In the inertial-polar chart, the wave operator acting on a function $\Phi: \mathbf{R}^{3} \rightarrow \mathbf{R}$ takes the form

$$
\square \Phi=-\partial_{t}^{2} \Phi+\frac{1}{r} \partial_{r}\left(r \partial_{r} \Phi\right)+\frac{1}{r^{2}} \partial_{\phi}^{2} \Phi
$$

We now restrict attention to fields and sources which are invariant under the one-parameter isometry group $G$ generated by $\xi$. This is equivalent to requiring

$$
\xi(\Phi)=0=\xi(j) .
$$

These conditions imply that $\Phi$ and $j$ define functions on the manifold of orbits $\mathbf{R}^{3} / G$, which shall be denoted by $\Psi$ and $f$, respectively. Because the source $j$ is assumed $G$-invariant, and because $G$ is an isometry group for the space-time, the wave equation (2.6) admits $G$ as a symmetry group and it descends to define a PDE relating $\Psi$ to $f$ on $\mathbf{R}^{3} / G \cdot{ }^{11,12}$ To obtain this differential equation in local coordinates we proceed as follows. Group invariants on $\mathbf{R}^{3}$ are functions of $r$ and

$$
\varphi:=\phi-\Omega t
$$

which define polar coordinates on $\mathbf{R}^{3} / G \approx \mathbf{R}^{2}$. In particular, granted (2.8), we have

$$
\Phi(t, r, \phi)=\Psi(r, \varphi), \quad j(t, r, \phi)=f(r, \varphi) .
$$

The reduced field equation on $\mathbf{R}^{3} / G$ can be obtained by substituting (2.10) into (2.6), which gives

$$
\frac{1}{r} \partial_{r}\left(r \partial_{r} \Psi\right)+\frac{1}{r^{2}} \chi(r) \partial_{\varphi}^{2} \Psi=f,
$$

where

$$
\chi(r)=1-\Omega^{2} r^{2}
$$

Note that the light cylinder on $\mathbf{R}^{3}$ projects to a light circle at $r=1 / \Omega$ on $\mathbf{R}^{3} / G$. Evidently, (2.11) is elliptic inside the light circle and hyperbolic outside the light circle, which is a consequence of the changing character (2.5) of the Killing vector $\xi$. Thus (2.11) is a PDE of mixed type.

In Ref. 7 (2.11) is solved on a disk of radius $R$ with source $f$ corresponding to two equal and opposite "scalar point charges" placed symmetrically relative to the origin. Sommerfeld conditions are imposed at the boundary of the disk, and it is required that $\Psi$ vanishes at the origin. The solution is given as a formal infinite series. As noted in Ref. 7, despite the mixed type of the PDE and, in particular, despite the fact that a Sommerfeld condition was enforced in the region where the PDE is hyperbolic, a unique solution exists. Here we provide a somewhat more general version of this result using the theory of symmetric positive equations. We consider (2.11) on a domain ${ }^{13}$ $\epsilon \leqslant r \leqslant R, 0<\epsilon<1 / \Omega$. We allow for a general source $f$ and we employ a class of boundary conditions that include Sommerfeld conditions at $r=R$, such as considered in Ref. 7. Specifically, we will impose the following boundary conditions:

$$
\Psi(\epsilon, \varphi)=0, \quad \tau(\varphi) R \partial_{r} \Psi(R, \varphi)+\sigma(\varphi) \partial_{\varphi} \Psi(R, \varphi)=0, \quad \sigma \tau \neq 0
$$

where $\sigma$ and $\tau$ represent smooth functions on the outer boundary $r=R$. Sommerfeld boundary conditions correspond to setting $\tau=1 / R$ and $\sigma= \pm \Omega$. 
Although we explicitly consider homogeneous boundary conditions (2.13), because we allow for an arbitrary source $f$ in (2.11) a large class of inhomogeneous boundary conditions

$$
\Psi(\epsilon, \varphi)=k(\varphi), \quad \tau(\varphi) R \partial_{r} \Psi(R, \varphi)+\sigma(\varphi) \partial_{\varphi} \Psi(R, \varphi)=l(\varphi),
$$

can also be accommodated. This is done by choosing a smooth function $\Lambda=\Lambda(r, \varphi)$ which satisfies the inhomogeneous boundary conditions (2.14) and then redefining

$$
\Psi \rightarrow \widetilde{\Psi}=\Psi-\Lambda, \quad f \rightarrow \widetilde{f}=f-\frac{1}{r} \partial_{r}\left(r \partial_{r} \Lambda\right)+\frac{1}{r^{2}} \chi(r) \partial_{\varphi}^{2} \Lambda
$$

$\widetilde{\Psi}$ now satisfies (2.11) with source $\widetilde{f}$ and homogeneous boundary conditions (2.13), to which our results apply.

\section{SYMMETRIC-POSITIVE PDEs}

Existence and uniqueness results can be obtained for linear PDEs of mixed type if they can be cast into first-order, symmetric-positive form with appropriate boundary conditions. ${ }^{9,10}$ Here we summarize the results from Refs. 9 and 10 which we shall need.

For our purposes, the data used to define a symmetric positive system of equations with admissible boundary conditions will be taken to be ${ }^{14}$

(i) a smooth manifold $M$ with smooth boundary $\partial M$; we set $\bar{M}=M \cup \partial M$;

(ii) a smooth scalar density of weight-1 on $M$, denoted by $\omega$; and

(iii) a finite-dimensional real vector space $V$ with scalar product $(\cdot, \cdot)$.

Let $u: \bar{M} \rightarrow V$. We consider a first-order system of differential equations for $u$ on $M$ of the form

$$
L u \equiv A^{a} \nabla_{a} u+B u=h,
$$

where $h: \bar{M} \rightarrow V$ and, at each $x \in M, A^{a}$ and $B$ are linear transformations:

$$
A^{a}(x): T_{x}^{*} M \times V \rightarrow V, \quad B(x): V \rightarrow V .
$$

For simplicity we assume that $A^{a}$ and $B$ depend smoothly on $x \in M$. The differential operator $\nabla_{a}$ is the exterior derivative on functions defined by any basis for $V$.

Definition 3.1: The system (3.1) is symmetric-positive if (1) $A^{a}$ defines a symmetric operator (with respect to the scalar product on $V$ ),

$$
\left(A^{a}(x) v_{a}\right)^{*}=A^{a}(x) v_{a}, \quad \forall v \in T_{x}^{*} M \quad \text { and } \forall x \in M
$$

and (2) the linear operator $K(x): V \rightarrow V$, defined by

$$
K=B-\frac{1}{2} \nabla_{a}\left(\omega A^{a}\right),
$$

has a positive-definite symmetric part:

$$
K(x)+K^{*}(x)>0, \quad \forall x \in M .
$$

We remark that $\nabla_{a}$ in (3.4) is defined by its unique torsion-free extension to vector densities of weight-1 on $M$ taking values in $V \otimes V^{*}$.

A class of boundary conditions on $u: \bar{M} \rightarrow V$ has been determined such that there exist unique solutions to symmetric-positive systems of PDEs. Following Friedrichs, we call these boundary conditions admissible. They are defined as follows.

Definition 3.2: Boundary conditions on $u: \bar{M} \rightarrow V$ are the requirement that, at each $x$ $\in \partial M, u(x)$ takes values in a linear subspace $N(x) \subset V$, which varies smoothly with $x$. 
We fix an outwardly oriented normal one-form, $n_{a}$, to the boundary $\partial M$. This one-form is uniquely determined up to multiplication by a smooth positive function on the boundary. We define $^{15}$

$$
\beta=\left.n_{a} A^{a}\right|_{\partial M} .
$$

Definition 3.3: Let $u: \bar{M} \rightarrow V$ be subject to the system of equations (3.1). The boundary conditions $u(x) \in N(x)$ on $\partial M$ are admissible if $N(x)$ is a maximal subspace such that the quadratic form $u \rightarrow(u, \beta u)$ is non-negative at each $x \in \partial M$.

Note that the admissibility of a set of boundary conditions does not depend upon the specific choice of covariant normal to the boundary. In the sequel we will make use of the following convenient characterization of admissible boundary conditions, which is due to Friedrichs. ${ }^{9}$

Proposition 3.4: Admissible boundary conditions, $u(x) \in N(x)$ on $\partial M$, are equivalent to the linear boundary conditions $\beta_{2} u=0$ on $\partial M$, where $\beta_{2}$ arises from a decomposition

$$
\beta=\beta_{1}+\beta_{2}
$$

such that, for all $x \in \partial M,(i)$ every $v \in V$ can be decomposed via

$$
v=v_{1}+v_{2}, \quad \beta_{1} v_{2}=\beta_{2} v_{1}=0,
$$

and (ii)

$$
\mu:=\beta_{1}-\beta_{2}
$$

has a non-negative symmetric part:

$$
\mu+\mu^{*} \geqslant 0 .
$$

We now summarize the existence and uniqueness results of Refs. 9 and 10 for symmetricpositive systems. We say that a mapping $u: M \rightarrow V$ is in $L^{2}(M, V)$ if

$$
\|u\|^{2} \equiv \int_{M}(u, u) \omega<\infty .
$$

Definition 3.5: Let the mappings $u: M \rightarrow V$ and $h: M \rightarrow V$ be in $L^{2}(M, V)$; $u$ is a strong solution to (3.1) satisfying the boundary conditions $u(x) \in N(x)$ on $\partial M$ if there exists a sequence of functions $\left\{u_{k}\right\} \in C^{\infty}(\bar{M}, V)$, satisfying the boundary conditions $u_{k}(x) \in N(x)$ on $\partial M$, such that

$$
u_{k} \rightarrow u, \quad \text { and } L u_{k} \rightarrow h
$$

in the $L^{2}$ (semi-)norm (3.11).

Theorem 3.6 (Friedrichs, Lax and Phillips): If (3.1) is symmetric-positive, then it admits a unique, strong solution satisfying admissible boundary conditions.

We remark that the theorems appearing in Refs. 9 and 10 use (piecewise) continuously differentiable functions $u_{k}$ to define strong solutions. However, it is straightforward to check that the relevant results go through for $\left\{u_{k}\right\} \in C^{\infty}(\bar{M}, V)$, which we use here.

\section{A SYMMETRIC-POSITIVE SYSTEM FOR THE HELICALLY-REDUCED WAVE EQUATION}

Here we show that the helically-reduced wave equation can be expressed in symmetricpositive form. We choose $M$ to be the annulus $M=\{(r, \varphi) \mid \epsilon<r<R\}$ equipped with the metric

$$
g=d r \otimes d r+r^{2} d \varphi \otimes d \varphi
$$


and associated volume density:

$$
\omega=(\operatorname{det} g)^{1 / 2}=r .
$$

We set $V=\mathbf{R}^{2}$ and equip it with the standard scalar product

$$
(u, v)=u_{1} v_{1}+u_{2} v_{2}
$$

We consider the following first-order system:

$$
\begin{aligned}
& \frac{1}{r} \partial_{r} u_{2}+\frac{1}{r^{2}} \chi \partial_{\varphi} u_{1}=f, \\
& \frac{1}{r} \partial_{r} u_{1}-\frac{1}{r^{2}} \partial_{\varphi} u_{2}=0 .
\end{aligned}
$$

Setting

$$
u_{1}=\partial_{\varphi} \Psi, \quad u_{2}=r \partial_{r} \Psi
$$

all classical $\left(C^{2}\right)$ solutions to (2.11) and (2.13) are solutions to (4.4) and (4.5). The solution $u$ thus obtained satisfies the boundary conditions

$$
u_{1}(\epsilon, \varphi)=0, \quad \sigma(\varphi) u_{1}(R, \varphi)+\tau(\varphi) u_{2}(R, \varphi)=0, \quad \sigma \tau \neq 0
$$

Conversely, given a classical $\left(C^{1}\right)$ solution to (4.4) and (4.5) satisfying boundary conditions (4.7), the $\left(C^{2}\right)$ function $\Psi$ defined by

$$
\Psi(r, \varphi)=\int_{\epsilon}^{r} d r^{\prime} \frac{1}{r^{\prime}} u_{2}\left(r^{\prime}, \varphi\right)
$$

satisfies (4.6) and hence (2.11) and (2.13). In this sense the equations (2.11) and (2.13) are equivalent to (4.4), (4.5), and (4.7). We write the system (4.4) and (4.5) as

$$
\left(\tilde{A}^{a} \nabla_{a} u-\tilde{h}\right)=0
$$

where

$$
\tilde{A}^{r}=\frac{1}{r}\left(\begin{array}{ll}
0 & 1 \\
1 & 0
\end{array}\right), \quad \tilde{A}^{\varphi}=\frac{1}{r^{2}}\left(\begin{array}{cc}
\chi & 0 \\
0 & -1
\end{array}\right), \quad \tilde{h}=\left(\begin{array}{l}
f \\
0
\end{array}\right),
$$

and

$$
u=\left(\begin{array}{l}
u_{1} \\
u_{2}
\end{array}\right)
$$

Now consider the following first-order system,

$$
L\left(\tilde{A}^{a} \nabla_{a} u-\tilde{h}\right)=0,
$$

where

$$
L=\left(\begin{array}{cc}
a & -c \chi \\
c & a
\end{array}\right)
$$


and $a=a(r)$ and $c=c(r)$ are smooth functions on $\bar{M}$. We require that $a$ and $c$ satisfy

$$
a^{2}+c^{2} \chi \neq 0
$$

so that (4.12) is equivalent to (4.9). We write (4.12) as

$$
A^{a} \nabla_{a} u=h,
$$

where

$$
A^{r}=\frac{1}{r}\left(\begin{array}{cc}
-c \chi & a \\
a & c
\end{array}\right), \quad A^{\varphi}=\frac{1}{r^{2}}\left(\begin{array}{cc}
a \chi & c \chi \\
c \chi & -a
\end{array}\right)
$$

and

$$
h=\left(\begin{array}{c}
a f \\
c f
\end{array}\right) .
$$

We now show that the functions $a$ and $c$ can be chosen so that the system (4.15) is symmetricpositive. The linear transformations defined by $A^{a}$ are symmetric for any choice of $a$ and $c$. Using (3.4) we find that

$$
K=K^{*}=\frac{1}{2}\left(\begin{array}{cc}
\partial_{r}(c \chi) & -\partial_{r} a \\
-\partial_{r} a & -\partial_{r} c
\end{array}\right) .
$$

Necessary and sufficient conditions for (3.5) are

$$
\partial_{r} c<0, \quad\left(\partial_{r} c\right) \partial_{r}(c \chi)<-\left(\partial_{r} a\right)^{2} .
$$

A pair of smooth functions that satisfy (4.19) and (4.14) for $0<\epsilon \leqslant r \leqslant R$ are of the form

$$
a=\text { const }, \quad c=-\alpha+e^{-\Omega^{3} r^{3}},
$$

provided the constants $|a|$ and $\alpha>0$ are chosen large enough, as is easily verified from

$$
\partial_{r} c=-3 \Omega^{3} r^{2} e^{-\Omega^{3} r^{3}}, \quad \partial_{r}(c \chi)=\Omega^{2} r\left\{2 \alpha-e^{-\Omega^{3} r^{3}}(2+3 \Omega r \chi)\right\} .
$$

Thus, with these choices for $a$ and $c$, the equations (4.15) are symmetric-positive. We summarize the preceding discussion as follows.

Proposition 4.1: The equations (2.11) and (2.13) are equivalent to the symmetric-positive system (4.15) and (4.7).

\section{ADMISSIBLE BOUNDARY CONDITIONS}

We now consider the boundary conditions (4.7) for which we have the following result.

Proposition 5.1: The constants $a$ and $\alpha$ in (4.13) and (4.20) can be chosen so that the boundary conditions (4.7) for (4.15) are admissible.

Proof: For the normal one-form to the boundary we use

$$
n=\left\{\begin{array}{l}
R d r \quad \text { at } r=R, \\
-\epsilon d r \quad \text { at } r=\epsilon .
\end{array}\right.
$$

We then have 


$$
\beta(R)=\left(\begin{array}{cc}
-c(R) \chi(R) & a \\
a & c(R)
\end{array}\right), \quad \beta(\epsilon)=\left(\begin{array}{cc}
c(\epsilon) \chi(\epsilon) & -a \\
-a & -c(\epsilon)
\end{array}\right)
$$

We consider boundary conditions of the form

$$
\sigma u_{1}+\tau u_{2}=0 \quad \text { on } \partial M
$$

where

$$
\sigma=1, \quad \tau=0, \quad \text { at } r=\epsilon,
$$

and

$$
\sigma=\sigma(\varphi) \neq 0, \quad \tau=\tau(\varphi) \neq 0, \quad \text { at } r=R .
$$

Following Proposition 3.4, we define

$$
\beta_{1}= \pm N\left(\begin{array}{cc}
-\tau^{2} c \chi-\sigma \tau a & \sigma \tau c \chi+\sigma^{2} a \\
\tau^{2} a-\sigma \tau c & -\sigma \tau a+\sigma^{2} c
\end{array}\right), \quad \beta_{2}= \pm N\left(\begin{array}{cc}
-\sigma^{2} c \chi+\sigma \tau a & -\sigma \tau c \chi+\tau^{2} a \\
\sigma^{2} a+\sigma \tau c & \sigma \tau a+\tau^{2} c
\end{array}\right),
$$

where

$$
N=\frac{1}{\sigma^{2}+\tau^{2}}
$$

so that

$$
\beta=\beta_{1}+\beta_{2} .
$$

In (5.6) the plus/minus sign is to be used at the outer/inner boundary. The boundary conditions (5.3) are equivalent to

$$
\beta_{2} u=0 \text { on } \partial M
$$

At the inner boundary we have

$$
\frac{1}{2}\left(\mu+\mu^{*}\right)=\left(\begin{array}{cc}
-c(\epsilon) \chi(\epsilon) & 0 \\
0 & -c(\epsilon)
\end{array}\right)
$$

We choose $\alpha$ sufficiently large so that $c(\epsilon)<0$ and we assume the inner boundary is within the light circle so we have $\chi(\epsilon)>0$. Therefore $\left(\mu+\mu^{*}\right)$ is non-negative at the inner boundary; the inner boundary condition is admissible. At the outer boundary we have

$$
\frac{1}{2}\left(\mu+\mu^{*}\right)=N\left(\begin{array}{cc}
-2 \sigma \tau a+\left(\sigma^{2}-\tau^{2}\right) c \chi & -\sigma \tau \Omega^{2} r^{2} c \\
-\sigma \tau \Omega^{2} r^{2} c & -2 \sigma \tau a+\left(\sigma^{2}-\tau^{2}\right) c
\end{array}\right)_{r=R} .
$$

Evidently, the outer boundary conditions are admissible in the case $\sigma \tau>0$ provided $a$ is chosen sufficiently negative. ${ }^{16}$ If $\sigma \tau<0$, the boundary conditions are admissible provided $a$ is chosen sufficiently positive. ${ }^{16}$ Thus the outer boundary conditions are admissible.

We remark that the admissibility of the outer boundary conditions did not depend upon the location of the outer boundary. Therefore, the outer boundary conditions can be imposed outside, inside, and even on the light circle. We also note that the proof of Proposition 5.1 shows that at the outer boundary neither Dirichlet conditions $(\sigma=1, \tau=0)$ nor Neumann conditions $(\sigma=0, \tau=1)$ for $\Psi$ are admissible. 


\section{CONCLUSIONS}

From Propositions 4.1 and 5.1 and Theorem 3.6 we have the following basic existence and uniqueness result for the helically-reduced wave equation in its symmetric positive form.

Theorem 6.1: The system (4.15) admits a unique, strong solution satisfying the boundary conditions (4.7).

While this theorem only establishes existence of distributional solutions to the first-order form of (2.11) and (2.13), it does imply that classical solutions to (2.11) and (2.13) are unique. Considerable additional analysis is needed to establish existence of $C^{2}$ solutions to (2.11) and (2.13). However, using Theorem 6.1 the following regularity properties of $\Psi$ can be immediately inferred.

Let us define $\widetilde{H}^{1}(M)$ as the completion in the $H^{1}$ norm,

$$
\|\Psi\|_{1}^{2}=\int_{M}\left(\Psi^{2}+g^{a b} \nabla_{a} \Psi \nabla_{b} \Psi\right) \omega
$$

of the space of smooth functions $\Psi$ satisfying the boundary conditions (2.13).

We can then deduce the following from Theorem 6.1.

Corollary 6.2: Let $u$ be the strong solution to (4.15) and (4.7). The function $\Psi$, defined by

$$
\Psi=\int_{\epsilon}^{r} d r^{\prime} \frac{1}{r^{\prime}} u_{2}\left(r^{\prime}\right) \equiv I(u)
$$

is in $\widetilde{H}^{1}(M)$ with (distributional) derivatives given by

$$
u_{1}=\partial_{\varphi} \Psi, \quad u_{2}=r \partial_{r} \Psi
$$

Proof: $I$ is easily verified to be a bounded linear transformation from the dense subspace $C^{\infty}(M, V) \subset L^{2}(M, V)$ to $L^{2}(M)$, so $I$ can be extended to all of $L^{2}(M, V)$ thus defining $\Psi$ $\in L^{2}(M)$ via (6.2). Let $u_{n}: M \rightarrow V$ be a sequence of smooth maps that converges to the strong solution $u$. Clearly,

$$
\left.I\left(u_{2 n}\right)\right|_{r=\epsilon}=0,
$$

so that the inner boundary condition is satisfied. We have

$$
\partial_{r} I\left(u_{2 n}\right)=\frac{1}{r} u_{2 n} \rightarrow \frac{1}{r} u_{2} \in L^{2}(M, V),
$$

and it is straightforward to verify that

$$
\partial_{\varphi} I\left(u_{2} n\right)=I\left(\partial_{\varphi} u_{2 n}\right) \rightarrow u_{1} \in L^{2}(M, V) .
$$

Thus $\Psi$ and its first derivatives are in $L^{2}$ and satisfy

$$
u_{1}=\partial_{\varphi} \Psi, \quad u_{2}=r \partial_{r} \Psi
$$

so that the outer boundary conditions are satisfied as well.

Physically, the source $f$ in (2.11) cannot be known with perfect precision. Furthermore, one may only have an approximately correct source appearing in a numerical solution. It is therefore important to note that the solution to (4.15) and (4.7) depends continuously upon the source $h$, so small changes/errors in the choice of $h$ lead to correspondingly small changes in the solution $u$ (or $\Psi)$. (Here "small" is defined by the $L^{2}$ norm.) To see this, we define a linear mapping $\mathcal{S}: L^{2}(M, V) \rightarrow L^{2}(M, V)$ that associates a solution $u=\mathcal{S}(h)$ of (4.15) and (4.7) to each source $h$. The existence of this mapping follows from Theorem 6.1. The uniqueness of strong solutions to 
(4.15) and (4.7) implies that the mapping $\mathcal{S}$ is closed and hence continuous by the closed graph theorem (see, e.g., Ref. 17). From Corollary 6.2 the mapping $\mathcal{I}: L^{2}(M, V) \rightarrow \widetilde{H}^{1}(M)$ is boundedhence continuous - and we then have the following corollary.

Corollary 4.3: $\Psi \in \tilde{H}^{1}(M)$ defined by (4.8) depends continuously upon the source $f$ $\in L^{2}(M)$.

\section{ACKNOWLEDGMENTS}

Thanks to Chris Beetle for influential discussions and for digging up Ref. 9. This work was supported in part by NSF Grant No. PHY-0070867 to Utah State University.

${ }^{1}$ R. Courant and D. Hilbert, Methods of Mathematical Physics, Vol. 2 (Interscience, New York, 1962).

${ }^{2}$ M. Taylor, Partial Differential Equations (Springer, New York, 1996).

${ }^{3}$ For a survey of results on differential equations of mixed type, see, e.g., M. Smirnov, Equations of Mixed Type (American Mathematical Society, Providence, RI, 1978); J. Stewart, Class. Quantum Grav. 18, 4983 (2001).

${ }^{4}$ J. Blackburn and S. Detweiler, Phys. Rev. D 46, 2318 (1992).

${ }^{5}$ J. Whelan, C. Beetle, W. Landry, and R. Price, Class. Quantum Grav. 19, 1285 (2002).

${ }^{6}$ J. Friedman, K. Uryu, and M. Shibata, Phys. Rev. D 6, 064035 (2002).

${ }^{7}$ J. Whelan, W. Krivan, and R. Price, Class. Quantum Grav. 17, 4895 (2000).

${ }^{8}$ It should be noted that one of the goals in Refs. 7 and 5 is to replace Sommerfeld conditions with "minimum energy radiation balanced" boundary conditions, which are nonlocal. It would appear that, generally speaking, nothing is known about the use of such boundary conditions with symmetric-positive equations.

${ }^{9}$ K. O. Friedrichs, Commun. Pure Appl. Math. 11, 333 (1958).

${ }^{10}$ P. Lax and R. Phillips, Commun. Pure Appl. Math. 13, 427 (1960).

${ }^{11}$ P. Olver, Applications of Lie Groups to Differential Equations (Springer-Verlag, New York, 1993).

${ }^{12}$ I. Anderson, M. Fels, and C. Torre, Commun. Math. Phys. 212, 653 (2000).

${ }^{13}$ The restriction to $\epsilon>0$ is needed to cast the field equations (2.11) into symmetric positive form.

${ }^{14}$ More general formulations are possible. For example, symmetric-positive equations can be defined for sections of a vector bundle. See Ref. 9.

${ }^{15}$ This definition can be made more explicit by extending $n_{a}$ in an arbitrary fashion into $M$, then defining $\beta(x)$ as the limit of the smooth family of linear transformations $n_{a}(x) A^{a}(x)$ as $x$ approaches $\partial M$. The result is independent of the extension of $n_{a}$ into $M$.

${ }^{16}$ Recall that the equations (4.15) are symmetric-positive provided $|a|$ is sufficiently large.

${ }^{17}$ M. Reed and B. Simon, Methods of Modern Mathematical Physics, Vol. I (Academic, New York, 1980). 